\title{
DERECHO Y POLÍTICAS AMBIENTALES EN ARAGÓN (SEGUNDO SEMESTRE 2020)
}

\author{
Olga HerRálz SerRano(*) \\ Letrada de las Cortes de Aragón \\ Profesora Asociada Doctora de Derecho Administrativo de la \\ Universidad de Zaragoza
}

(*) El presente trabajo se incluye dentro de las actividades del Grupo consolidado de investigación ADESTER (Estudios Territoriales), reconocido por el Gobierno de Aragón (BOA núm. 48, de 20 de abril de 2005). 
Sumario: 1. INTRODUCCIÓN. 2. LEGISLACIÓN: 2.1. Caza. 2.2. Trashumancia. 2.3. Tributos ambientales. 2.4. Emisión de gases de efecto invernadero. 2.5. Incendios forestales. 3 ORGANIZACIÓN: LA RENOVACIÓN DE LA COMPOSICIÓN DE DETERMINADOS ÓRGANOS DE PARTICIPACIÓN SOCIAL EN MATERIAS AMBIENTALES. 4. JURISPRUDENCIA: EL TRIBUNAL SUPERIOR DE JUSTICIA DE ARAGÓN ANULA EL PLAN DE PROTECCIÓN DEL PAISAJE PROTEGIDO DE LOS PINARES DE RODENO QUE ESTABA LLAMADO A SUSTITUIR EL PRUG QUE FUE ANULADO POR EL MISMO MOTIVO POR EL TRIBUNAL SUPREMO. 5. MEDIDAS DE FOMENTO DE CARÁCTER AMBIENTAL.

\section{INTRODUCCIÓN}

Marcado el año 2020 por la crisis sanitaria ocasionada por la pandemia del COVID-19 y sus repercusiones en todas las políticas sectoriales de todos los Gobiernos, el segundo semestre que analizamos aquí se incardina igualmente en ese contexto donde casi todo ha sido secundario respecto de los esfuerzos principales por controlar la propagación del virus y minimizar el impacto económico y social que la pandemia está teniendo. De ahí que muchas de las escasas novedades legislativas en materia medioambiental que se han producido en la Comunidad Autónoma de Aragón sean de rango inferior y se encuentren motivadas por el impacto del COVID-19 en el sector. Nos referiremos a ella en el apartado siguiente, pero antes daremos cuenta de la polémica sostenida por Aragón con la Administración del Estado en materia hídrica, a propósito de una transferencia de aguas del río Ebro a Cantabria.

Con fecha 9 de marzo de 2020, se recibió de la Dirección General del Agua del Ministerio para la Transición Ecológica y el Reto Demográfico solicitud de emisión de informe preceptivo de la Comunidad Autónoma de Aragón sobre la propuesta de Acuerdo de Consejo de Ministros para autorizar una transferencia anual de carácter indefinido de 4,99 $\mathrm{hm}^{3}$ de recursos hídricos desde la Demarcación Hidrográfica del Ebro a la Demarcación Hidrográfica del Cantábrico Occidental, para necesidades de abastecimiento de determinadas comarcas de Cantabria.

Al respecto, se siguió el procedimiento establecido en la disposición adicional primera de la Ley de Aguas y Ríos de Aragón según el cual, para cualquier propuesta de transferencia de aguas que afecte a los intereses de Aragón, en el marco de lo previsto en el artículo 72.3 del Estatuto de Autonomía, y en defensa de las competencias e intereses de la Comunidad, debían emitir informe, al menos, con la finalidad de conformar la decisión del Gobierno de Aragón, el Instituto Aragonés del Agua, el Consejo de Ordenación del Territorio, la Dirección General de los Servicios Jurídicos y el Consejo Consultivo de Aragón. La emisión de dichos informes se vio interrumpida por la suspensión de los plazos administrativos establecida en el Real Decreto 463/2020, de 14 de marzo, por el que se declaró en España el estado de alarma. De ahí que no pudiéramos conocer su contenido hasta el mes de junio, culminándose el procedimiento 
recogido en la citada disposición adicional primera de la Ley de Aguas y Ríos de Aragón en el mes de julio.

Hasta el momento de la solicitud de informe por parte de la Administración del Estado, se encontraban vigentes dos "bitrasvases" previos entre las demarcaciones hidrográficas del Ebro y del Cantábrico Occidental. En ambos casos, los recursos hídricos pasan, en períodos "húmedos", de la segunda demarcación a la primera (aprovechando su almacenamiento en el embalse del Ebro) para, correlativamente, retornar, en períodos "secos", a su demarcación hidrográfica de origen, equilibrándose el balance hídrico teóricamente en períodos de 4 años. Uno de los bitrasvases, denominado "Ebro-Besaya", data de 1982 mientras el llamado "Ebro-Besaya-Pas" estuvo vigente entre 2008 y 2015, fecha en que fue anulado judicialmente, estando pendiente de sustitución por otro en tramitación.

Así las cosas, en ese contexto, resultó determinante para los distintos órganos informantes de la Comunidad Autónoma de Aragón la suficiencia o no de esos dos bitrasvases para satisfacer las demandas de abastecimiento que se alegaban como justificativas de la propuesta de transferencia anual de carácter indefinido de 4,99 hm 3 de agua.

El informe emitido por la Dirección General de Servicios Jurídicos, de fecha 15 de junio de 2020, hace un pormenorizado recorrido por las condiciones y requisitos previstos y concluye que se cumplen las condiciones ambientales, el destino de la transferencia al abastecimiento, el principio de recuperación de costes y el principio de garantía de las demandas actuales y futuras de todos los usos y aprovechamientos de la cuenca cedente, de conformidad con los artículos 12 y siguientes de la Ley 10/2001, de 5 de julio, del Plan Hidrológico Nacional. A ello se añade que tampoco los informes del Instituto Aragonés del Agua (de 3 de abril) ni del Consejo de Ordenación del Territorio (de 9 de junio) plantean objeciones sobre la cuestión ambiental. Sin embargo, todos los informes contienen un reparo de carácter jurídico relativo a la deficiente motivación de la transferencia de recursos hídricos que contiene la propuesta del acuerdo del Consejo de Ministros. En particular, el Dictamen 103/2020, de 30 de junio, del Consejo Consultivo de Aragón considera que el órgano estatal no ha justificado la necesidad de recursos hídricos que permita conocer cuál es el déficit exacto de agua que motiva la adopción de la transferencia y si esa necesidad es nueva y diferente o si implica un déficit de recursos hídricos mayor que el que se cubría con los bitrasvases aplicados en los últimos años. Seguía diciendo el Consejo Consultivo que, aunque se diesen las condiciones previstas en la LPHN y a pesar de que los volúmenes a transferir en la autorización propuesta representasen una cantidad "exigua" respecto del agua almacenada en el embalse del Ebro, faltaba una explicación acabada de las 
características del déficit hídrico que se pretendía atajar dado el carácter de "solución última y más comprometida" con que la ley configura este tipo de instrumentos de planificación hidrológica.

"A todo ello debemos añadir que tampoco se ofrece explicación alguna de las razones por las que se autoriza la transferencia de pequeña cuantía "con carácter indefinido", cuando se advierte que, al mismo, tiempo, se está tramitando una nueva autorización especial para el bitrasvase Ebro-Besaya-Pas".

Aspecto este, el de la falta de justificación objetiva del carácter permanente e indefinido de la transferencia proyectada, que también subrayaron el Instituto Aragonés del Agua y el COTA para informarla negativamente. Si las transferencias de aguas entre cuencas deben reservarse como última solución a los problemas, los informes destacaron que era imprescindible motivar debidamente la necesidad no solo de la cantidad concreta de recursos hídricos afectados, sino también del período temporal al que iba referida la autorización y la insuficiencia de los trasvases reversibles que se encontraban en funcionamiento.

Esos "bitrasvases", según la disposición adicional sexta de la LPHN, quedan exceptuados de la aplicación del régimen jurídico previsto para las transferencias de aguas y, en consecuencia, de sus condiciones y requisitos pues, en sentido estricto, no suponen una transferencia de recursos hídricos, ya que el agua retorna a su cuenca de origen en un periodo de cuatro años, con la exigencia de un balance hídrico equilibrado. Equilibrio este que la propia Confederación Hidrográfica del Ebro reconoce que no se ha cumplido cuando en el procedimiento seguido para la actualización del segundo bitrasvase tras su anulación judicial afirmaba:

"En esta situación, la Confederación Hidrográfica del Ebro ha decidido eximir al Gobierno de Cantabria de la devolución de los volúmenes derivados en los ejercicios 2014 y anteriores (.,,), y comenzar de nuevo la cuenta de tiempo para alcanzar el plazo máximo de cuatro años para la devolución de los caudales detraídos" (BOE núm. 91, de 14 de abril de 2018).

Este dato no pasó desapercibido en el Dictamen 103/2020, del Consejo Consultivo de Aragón, que, aunque no plantea objeción alguna a la afirmación de que los volúmenes de agua transferidos a través de la infraestructura de los bitrasvases no hayan perjudicado las necesidades hídricas de la demarcación hidrográfica del Ebro, sí concluye que se han estado derivando recursos sin cumplir las condiciones marcadas por la disposición adicional sexta de la LPHN, que, siempre según sus palabras,

“establece unos requisitos muy restrictivos (el retorno de las aguas en un plazo máximo de cuatro años y el balance hídrico equilibrado) para exceptuar la aplicación del régimen jurídico previsto para las transferencias de aguas.

A la vista de las circunstancias de hecho y de derecho concurrentes, el Consejo Consultivo de .Aragón informa con carácter desfavorable la propuesta para autorizar una transferencia anual de 4,99 hm3 de recursos hídricos desde la Demarcación Hidrográfica del Ebro a la Demarcación Hidrográfica del Cantábrico Occidental para necesidades de abastecimiento de determinadas comarcas de Cantabria". 
Elevados a la consideración del Gobierno de Aragón los cuatro informes citados, este los hizo suyos para informar negativamente la propuesta de transferencia dado que no se justificaba la necesidad ni de la cantidad de agua solicitada ni del carácter permanente y no reversible de la misma, a la vista de la vigencia de los dos bitrasvases aludidos en los últimos años. Aunque los problemas puntuales de abastecimiento de agua de boca de Santander se han ido solventando con ellos, el Gobierno cántabro insistió en la necesidad de una transferencia permanente de aguas del Ebro, que contó no solo con el rechazo frontal del Gobierno de Aragón, sino también de las Cortes de Aragón en las que, por imperativo de la disposición adicional primera de la Ley de Aguas y Ríos, se debatió el informe del ejecutivo autonómico. El Pleno de las Cortes de Aragón, en sesión celebrada el 9 de julio de 2020, conoció el informe de rechazo enviado por el Gobierno, lo aprobó con "aparente" unanimidad y lo remitió a la Administración General del Estado. Decimos aparente porque el G.P. VOX advirtió con posterioridad que se había equivocado en la votación, pidiendo que constara en acta que su intención era abstenerse.

No obstante la oposición del Gobierno y de las Cortes de Aragón, el Consejo de Ministros, con los informes favorables de las dos demarcaciones hidrográficas implicadas, acabó autorizando la transferencia con fecha 4 de agosto de 2020.

\section{LEGISLACIÓN}

\subsection{CaZa}

El Decreto-Ley 1/2020, de 25 de marzo, del Gobierno de Aragón, por el que se adoptaron medidas urgentes para responder al impacto generado por el COVID-19 en la Comunidad Autónoma, declaró el carácter esencial de las actividades precisas para garantizar la cadena alimentaria durante la vigencia del estado de alarma en nuestro país, a la vez que admitía la continuidad de aquellos procedimientos requeridos por la gestión de la situación de crisis sanitaria ocasionada por el virus y sus repercusiones económicas y sociales. En ese contexto y ante las sucesivas prolongaciones temporales del estado de alarma, el Ministerio de Agricultura, Pesca y Alimentación instó a las Comunidades Autónomas a adoptar las medidas de control precisas en relación con aquellas especies cinegéticas que estuviesen generando riesgos y amenazas para la agricultura y la ganadería o en situación de desequilibrio poblacional.

A dichas finalidades respondió la Orden AGM/329/2020, de 17 de abril (BOA 20 abril), que autorizó en Aragón las actividades cinegéticas cuya exclusiva finalidad fuese el control poblacional del jabalí, conejo, corzo, ciervo y cabra montés. Sin embargo, dicha disposición fue derogada tan solo un mes después por Orden 
AGM/400/2020, de 20 de mayo (BOA 22 mayo), una vez que, en el marco del Plan para la transición hacia una nueva normalidad que el Consejo de Ministros aprobó el 28 de abril, el Ministerio de Sanidad (Orden SND/414/2020, de 16 de mayo) permitiera la actividad cinegética en todas sus modalidades y sin restricciones, siempre que se respetase la distancia de seguridad y las medidas de higiene y prevención fijadas por las autoridades sanitarias. Desde aquel momento dejó de ser necesario el control poblacional excepcional que fue objeto de la Orden AGM/329/2020, pudiendo ejercerse la actividad cinegética en el marco de la Ley de Caza de Aragón, el Plan General de Caza y demás disposiciones relacionadas.

\subsection{Trashumancia}

Aunque no constituya una práctica muy extendida, la trashumancia en Aragón ha articulado las relaciones entre la montaña y el llano, entre los Pirineos y el Valle del Ebro, así como entre las serranías ibéricas turolenses y Levante, La Mancha y Andalucía. De hecho, el Gobierno de Aragón declaró la trashumancia Bien de Interés Cultural Inmaterial (Decreto 289/2011, de 30 de agosto), lo que conlleva un deber genérico de protección de dicha práctica. En el ejercicio que analizamos, fue aprobada la Orden AGM/469/2020, de 8 de junio, por la que se establecen las condiciones y los requisitos necesarios para realizar la trashumancia del ganado vacuno desde el valle de Broto hasta los pastos comunales de la zona Gavarnie en Francia en el año 2020 (BOA 18 junio). Aunque ese traslado geográfico en concreto es una práctica ancestral que data de finales del s. XIX, ha conocido limitaciones con objeto de proteger dichos animales de enfermedades emergentes que hay en la Unión Europea, como la lengua azul.

De ahí que la Orden a que hacemos referencia regule tanto los aspectos en materia de sanidad animal como las obligaciones formales que deben observar los movimientos de ganados trashumantes en ese itinerario geográfico para asegurar la pervivencia de su práctica en condiciones de seguridad.

\subsection{TRIBUtos}

Siempre en respuesta a la situación originada por la pandemia del COVID-19 y, más en concreto, en este caso para hacer frente a la disminución en la actividad de determinados establecimientos comerciales, se aprobaron una serie de medidas tributarias llamadas a tener una duración limitada en el tiempo. Por cuanto afecta a los tributos ambientales, objeto de atención en el presente trabajo, la Ley 1/2020, de 9 de julio, por la que se establecen medidas excepcionales para el ejercicio 2020 en 
determinados tributos gestionados por la Comunidad Autónoma de Aragón (BOA 17 julio) previó una bonificación del 20 por ciento en el Impuesto Medioambiental sobre las grandes áreas de venta. La condición para aplicarla sobre la cuota tributaria es que se trate de establecimientos cuya parte de negocio dedicado a la venta minorista de productos de higiene y alimentación ocupe menos del $80 \%$ de su superficie o cuyo negocio no sea la venta minorista de tales productos, al haber sido los más golpeados por la suspensión de actividades durante la vigencia del estado de alarma.

\subsection{EMISIÓN DE GASES DE EFECTO INVERNADERO}

La Ley 1/2005, de 9 de marzo, por la que se regula el régimen del comercio de derechos de emisión de gases de efecto invernadero, transpuso al ordenamiento jurídico español la Directiva 2003/87/CE, del Parlamento Europeo y del Consejo, de 13 de octubre, que fue modificada con posterioridad por la Directiva (UE) 2018/410 del Parlamento Europeo y del Consejo, de 14 de marzo. La Ley 1/2005, de 9 de marzo, obliga a que toda instalación en la que se desarrolle alguna actividad de las especificadas en su anexo I, cuente con autorización de emisión de gases de efecto invernadero, salvo si la instalación está excluida del régimen comunitario con arreglo a lo regulado en su disposición adicional cuarta. Dicha autorización debe incluir un plan de seguimiento de emisiones de gases de efecto invernadero de la instalación.

En el ámbito de la Comunidad Autónoma de Aragón, mediante la Orden de 27 de agosto de 2012, del Consejero de Agricultura, Ganadería y Medio Ambiente, se reguló la solicitud de autorización de emisión de gases de efecto invernadero para el período 2013-2020. Esa autorización dejaba de tener vigencia el 31 de diciembre de 2020, y, por lo tanto, se hacía preciso aprobar una nueva, lo que tuvo lugar mediante la Orden AGM/759/2020, de 10 de agosto, por la que se regula la solicitud de autorización de emisión de gases de efecto invernadero para el período 2021-2030 (BOA 20 agosto). Esta disposición regula en la actualidad, por tanto, el plazo, contenido y modelo oficial de las solicitudes de autorización de emisión de gases de efecto invernadero, modificación o baja de la misma y las plantillas a utilizar en la elaboración del plan de seguimiento de emisiones de aquellas instalaciones ubicadas en la Comunidad Autónoma de Aragón que, durante el periodo 2021-2030, desarrollen actividades incluidas en el ámbito de aplicación de la Ley 1/2005.

\subsection{INCENDIOS FORESTALES}

El 29 de febrero entró en vigor la Orden AGM/139/2020, de 10 de febrero, por la que se prorrogaba transitoriamente la Orden de 20 de febrero de 2015, del Consejero de 
Agricultura, Ganadería y Medio Ambiente, sobre prevención y lucha contra los incendios forestales en la Comunidad Autónoma de Aragón para la campaña 2015/2016. En virtud de aquella Orden, se estableció como época de peligro de incendios forestales para el año 2020 el período comprendido entre el 1 de abril y el 15 de octubre, ambos incluidos. Las dimensiones alcanzadas por la pandemia del COVID-19 obligaron, no obstante, a regular de manera excepcional la realización de fuego fuera de dicho período y a prorrogar para determinados usos sin necesidad de tramitar nuevas comunicaciones o solicitudes de autorización por parte de los que ya hubieran dispuesto de ellas en la última anualidad o bien, en el caso de nuevos usuarios, presentándolas de forma telemática. Todo ello por una coyuntura ambiental favorable en virtud de la Orden AGM/975/2020, de 28 de septiembre (BOA 14 octubre) y de cara asimismo a evitar aquellos desplazamientos de la población que no se fuesen estrictamente indispensables.

\section{ORGANIZACIÓN: LA RENOVACIÓN DE LA COMPOSICIÓN DE DETERMINADOS ÓRGANOS DE PARTICIPACIÓN SOCIAL EN MATERIAS AMBIENTALES}

El artículo 36.1 de la Ley 10/2014, de 27 de noviembre, de Aguas y Ríos de Aragón, regula la composición de la Comisión del Agua de Aragón, órgano de participación social en la materia, distinguiendo entre representantes institucionales y de organizaciones sociales, asociaciones y usos diversos del agua. El citado precepto establece como representantes de las instituciones públicas con presencia en la Comisión del Agua de Aragón, a dos representantes de la Universidad de Zaragoza (apartado e), tres representantes de los municipios de Huesca, Teruel y Zaragoza (apartado g), tres representantes de las comarcas aragonesas (apartado i), cuatro representantes de la Administración de la Comunidad Autónoma de Aragón (apartado n), un representante designado por cada uno de los Grupos Parlamentarios de las Cortes de Aragón (apartado ñ) y un representante de la Confederación Hidrográfica del Ebro, otro de la del Tajo y otro de la del Júcar (apartado o).

Como tras las elecciones autonómicas y municipales de mayo de 2019 era preceptiva la renovación de la composición de la citada Comisión del Agua, las instituciones designaron a sus nuevos representantes y trasladaron dichas designaciones al Presidente del Instituto Aragonés del Agua a los efectos de su nombramiento, que tuvo lugar mediante Resolución de 1 de junio de 2020, por la que se efectúa la renovación y nombramiento de representantes institucionales y de expertos en materias hídricas en la Comisión del Agua de Aragón (BOA 9 junio). Asimismo, por medio de la Resolución de 5 de junio de 2020, del Presidente del Instituto Aragonés del Agua, se 
efectuó el nombramiento de los representantes renovados a propuesta de las siguientes organizaciones sociales: la Asociación de Entidades Locales del Pirineo Aragonés (ADELPA), la Federación Aragonesa de Municipios, Comarcas y Provincias, la Unión de Agricultores y Ganaderos de Aragón, la Asociación Agraria de Jóvenes Agricultores y la Confederación de Empresarios de Aragón (BOA 17 junio).

En otro orden de cosas, el ejercicio que analizamos también conoció el cese y nueva designación de Presidentes de otros órganos de participación social, los Patronatos de Espacios Naturales Protegidos, más en concreto del Parque Natural de la Sierra y Cañones de Guara, del Parque Natural de los Valles Occidentales, del Parque Natural de Posets Maladeta, del Parque Natural del Moncayo, de la Reserva Natural Dirigida de los Sotos y Galachos del Ebro, del Paisaje Protegido de los Pinares de Rodeno, del Paisaje Protegido de la Sierra de Santo Domingo, de los Monumentos Naturales del Maestrazgo, de los Monumentos Naturales de los Glaciares Pirenaicos, y de la Laguna de Gallocanta. Los respectivos Acuerdos adoptados por el Gobierno de Aragón en su reunión de 17 de junio de 2020 fueron publicitados mediante la Orden AGM/606/2020, de 30 de junio (BOA 16 julio).

\section{JURISPRUDENCIA: EL TRIBUNAL SUPERIOR DE JUSTICIA DE ARAGÓN ANULA EL PLAN DE PROTECCIÓN DEL PAISAJE PROTEGIDO DE LOS PINARES DE RODENO QUE ESTABA LLAMADO A SUSTITUIR EL PRUG QUE FUE ANULADO POR EL MISMO MOTIVO POR EL TRIBUNAL SUPREMO}

La Orden AGM/611/2020, de 22 de junio (BOA 17 julio), dispuso la publicación de la parte dispositiva de la Sentencia número 20/2020, de 13 de enero de 2020, dictada por la Sala de lo Contencioso-Administrativo del Tribunal Superior de Justicia de Aragón, Sección primera, declarando la nulidad del Decreto 187/2014, de 18 de noviembre, del Gobierno de Aragón, por el que se aprueba el Plan de Protección del Paisaje Protegido de los Pinares de Rodeno.

En efecto, para la gestión del Paisaje se aprobó en su día el Plan Rector de Uso y Gestión por Decreto 65/1998, de 15 de marzo, que fue anulado por STS de 10 de diciembre de 2009, debido a la falta de memoria económica que pudiera prever indemnizaciones por limitaciones a la propiedad privada. Aprobado el Plan de Protección del Paisaje protegido de los Pinares de Rodeno, como figura de gestión que estaba llamada a sustituir el PRUG anulado, mediante el referido Decreto 187/2014, este fue también impugnado por idéntico motivo de que, en su memoria económica, no se 
consignaba cantidad alguna para eventuales compensaciones a los propietarios de los terrenos por privaciones o limitaciones de uso. Y ello a pesar de que tanto el informe de la Dirección General del Medio Natural como el del Consejo de Ordenación del Territorio de Aragón ya habían puesto de manifiesto, en el procedimiento de aprobación del instrumento de gestión, que debería de constar partida para ello.

Los recurrentes, propietarios proindiviso de unas fincas rústicas incluidas en el ámbito de aplicación del Paisaje Protegido, adujeron que la memoria económica debía establecer una estimación aproximada en concepto de indemnizaciones y que no bastaba con prever el concepto con una consignación de cero euros para el mismo como hacía el Decreto impugnado. Como también señalaron los recurrentes, la cuestión había sido resuelta de forma reiterada por el Tribunal Supremo y así lo apreció la Sala de lo Contencioso-Administrativo del Tribunal Superior de Justicia de Aragón, que, en aplicación de dicha jurisprudencia, en su Sentencia núm. 20/2020 (JUR\2020172858), reprodujo la siguiente afirmación:

"En cuanto a la memoria económica, es cierto que no cabe exigir una ponderación detallada y exacta de todos los costes que pueda suponer el reglamento, pues se trata de datos cuya completa determinación puede resultar imposible en el momento de aprobarse aquél, pero al menos es preciso la elaboración de una estimación aproximada que tenga en cuenta las variables que puedan producirse".

El Tribunal asimismo rechazaba el argumento de la Administración de que tenían que ser los propietarios los que acreditaran la realidad de las limitaciones y su coste, recordando el deber de aquella de efectuar cuando menos una estimación de ello so pena, como era el caso, de recoger una memoria económica "vacía de contenido y sin trascendencia práctica alguna".

\section{MEDIDAS DE FOMENTO DE CARÁCTER AMBIENTAL}

Meramente, a título informativo de la política de fomento en materia medio ambiental seguida por la Administración de la Comunidad Autónoma durante el segundo semestre del año 2020, se convocaron subvenciones para el ejercicio en materia de: en materia de gestión de riesgos de inundación, para la redacción de planes municipales de actuación ante riesgos de inundación y la adquisición de equipos y medios materiales de protección frente a inundaciones (Orden AGM/447/2020, de 10 de marzo, BOA 10 junio); en el área de influencia socio-económica del Parque Nacional de Ordesa y Monte Perdido, de acuerdo con lo previsto en el Real Decreto 1229/2005, de 13 de octubre (Orden AGM/568/2020, de 18 de junio, BOA 8 julio); para la adaptación de la ganadería extensiva a los retos ambientales y a los desafíos socioterritoriales (Orden AGM/618/2020, de 10 de julio, BOA 21 julio); y las ayudas LEADER para la 
realización de operaciones conforme a las estrategias de desarrollo local LEADER (Orden AGM/929/2020, de 4 de septiembre, BOA 30 septiembre).

Asimismo por Orden AGM/241/2020, de 10 de marzo (BOA 23 marzo), se convocó el Premio Aragón Medio Ambiente para el año 2020, que fue otorgado por Acuerdo de 2 de septiembre de 2020, del Gobierno de Aragón (BOA 15 septiembre). 